\title{
Collective excitations of the QED vacuum
}

\author{
D. C. Ionescu* and W. Greiner \\ Institut für Theoretische Physik, J. W. Goethe Universität, Robert Mayer Strasse 8-10, \\ D-6000 Frankfurt am Main, Federal Republic of Germany \\ B. Müller \\ Physics Department, Duke University, Durham, North Carolina 27706 \\ G. Soff \\ Gesellschaft für Schwerionenforschung (GSI), Planckstraße 1, Postfach 110 552, \\ D-6100 Darmstadt, Federal Republic of Germany
}

(Received 8 June 1992)

\begin{abstract}
Using relativistic Green's-function techniques we examined single-electron excitations from the occupied Dirac sea in the presence of strong external fields. The energies of these excited states are determined taking into account the electron-electron interaction. We also evaluate relativistic transition strengths incorporating retardation, which represent a direct measure of correlation effects. The shifts in excitation energies are computed to be lower than $0.5 \%$, while the correlated transition strengths never deviate by more than $10 \%$ from their bare values. A major conclusion is that we found no evidence for collectivity in the electron-positron field around heavy and superheavy nuclei.

PACS number(s): 12.20. Ds
\end{abstract}

\section{INTRODUCTION}

In this paper we investigate the possible existence of collective excitations of the Dirac vacuum in the presence of a strong Coulomb field. We address the question of whether the QED ground state possesses excitation modes of a collective nature, which is a well-known topic of many-body theory [1].

As a physical system we consider a completely ionized quasiatom of charge $Z \leq 170$, which can be formed for a short period of time (of order of $10^{-21} \mathrm{~s}$ ) in collisions of very heavy ions. The solutions of the Dirac equation for stationary external fields lead to bound states with discrete energies in the energy gap $-m \leq E \leq m$. In addition, one obtains a spectrum of continuum wave functions with energies varying between $-\infty$ and $-m$ as well as between $m$ and $\infty$. For nuclear charge numbers exceeding the critical value $Z_{\mathrm{cr}} \simeq 170$, the lowest discrete energy state $(1 s)$ dives into the negative-energy continuum below $-m$ and loses its pure bound-state character. It becomes a resonance imbedded into the continuum. Such an unoccupied bound state opens the possibility of spontaneous positron creation [2,3] and the QED ground state becomes charged [4].

However, for nuclear charge numbers $Z \leq Z_{\text {cr }}$ the energy of the $1 s$ state is just above the negative-energy continuum which is completely occupied according to Dirac's hole theory. Electron excitations from these negativeenergy states below $-m$ into the empty bound states above the Fermi level lead to interacting particle $\left(e^{-}\right)$hole $\left(e^{+}\right)$states which in principle allow for collective excitation modes. This may correspond to macroscopic density fluctuations. According to traditional manybody theory we interpret the transition to a collective excited state as the creation of a quasiparticle representing a coherent superposition of many particle-hole states $[5,6]$. Collective excitations have been studied in connection with longitudinal waves in plasmas, x-ray absorption in metals, zero sound in dense systems, giant resonances in photonuclear reactions, etc. Several years ago it was reported [7] that there is evidence for the existence of collective excitations of the Dirac vacuum in strong Coulomb fields. A collective excitation is caused by the interaction, or, more accurately, by the correlation between particles. If this interaction is turned off, a collective excited state dissociates into individual-particle excited states.

The major theoretical ingredients of our treatment are presented in Sec. II, where the fundamental equations are derived. The application of the Bethe-Salpeter equation to collective excitations is presented in Sec. III. The general formulas are reduced to a form suitable for the numerical analysis in Sec. IV. Sections V and VI contain the discussion of our results and the conclusions, respectively.

\section{FORMULATION OF THE PROBLEM}

Our starting point is the four-point function which is defined by [8]

$K\left(x_{1}, x_{2}, x_{3}, x_{4}\right)=\left\langle\Phi\left|T \hat{\psi}\left(x_{1}\right) \hat{\psi}\left(x_{3}\right) \hat{\psi}^{\dagger}\left(x_{2}\right) \hat{\psi}^{\dagger}\left(x_{4}\right)\right| \Phi\right\rangle$,

where $|\Phi\rangle$ represents the ground state of the interacting system, $\hat{\psi}(x)$ and $\hat{\psi}^{\dagger}(x)$ are fermion-field operators in the Heisenberg picture, and $T$ denotes the time-ordering 
operator. The arguments of the field operators in the above definition denote space-time coordinates and the quantum numbers which are appropriate for the considered system. $K\left(x_{1}, x_{2}, x_{3}, x_{4}\right)$ is of course unknown due to the fact that we do not know the exact ground state of the interacting system. The function $K$ describes the propagation of two particles, or two holes, or of one par- ticle and one hole. As we regard a collective excitation as a quasiparticle consisting of a particle and a hole, we choose $t_{2}>t_{1}$ and $t_{4}>t_{3}$ such that $K$ has the general structure $\left\langle\psi^{\dagger} \psi \psi^{\dagger} \psi\right\rangle$, describing the $p$ - $h$ propagation. In a time-independent external field the function $K$ depends only on time differences and with $\tau=t_{1}-t_{3}$ we obtain from Eq. (1)

$K\left(\mathbf{r}_{1}, \mathbf{r}_{2}, \mathbf{r}_{3}, \mathbf{r}_{4} ; \tau\right)=-\theta(\tau)\left\langle\Phi\left|\hat{\psi}^{\dagger}\left(\mathbf{r}_{2}\right) \hat{\psi}\left(\mathbf{r}_{1}\right) e^{-i\left(\hat{H}-E_{0}\right) \tau} \hat{\psi}^{\dagger}\left(\mathbf{r}_{4}\right) \hat{\psi}\left(\mathbf{r}_{3}\right)\right| \Phi\right\rangle-\theta(-\tau)\left\langle\Phi\left|\hat{\psi}^{\dagger}\left(\mathbf{r}_{4}\right) \hat{\psi}\left(\mathbf{r}_{3}\right) e^{i\left(\hat{H}-E_{0}\right) \tau} \hat{\psi}^{\dagger}\left(\mathbf{r}_{2}\right) \hat{\psi}\left(\mathbf{r}_{1}\right)\right| \Phi\right\rangle$

where the fermion-field operators became time independent according to the relation $\hat{\psi}(\mathbf{r}, t)=e^{i \hat{H} t} \hat{\psi}(\mathbf{r}) e^{-i \hat{H} t}$. We denote the ground-state energy of the interacting system by $E_{0}$. We define the Fourier transform of this function by $K(\omega)$

$$
K(\omega)=\int_{-\infty}^{\infty} d \tau e^{i \omega \tau} K(\tau)
$$

and with Eq. (2) we obtain the Lehmann representation of the four-point function $K$ :

$$
K(\omega)=\left\langle\Phi\left|\hat{\psi}^{\dagger}\left(\mathbf{r}_{4}\right) \hat{\psi}\left(\mathbf{r}_{3}\right) \frac{i}{\omega+\hat{H}-E_{0}-i \delta} \hat{\psi}^{\dagger}\left(\mathbf{r}_{2}\right) \hat{\psi}\left(\mathbf{r}_{1}\right)\right| \Phi\right\rangle-\left\langle\Phi\left|\hat{\psi}^{\dagger}\left(\mathbf{r}_{2}\right) \hat{\psi}\left(\mathbf{r}_{1}\right) \frac{i}{\omega-\hat{H}+E_{0}+i \delta} \hat{\psi}^{\dagger}\left(\mathbf{r}_{4}\right) \hat{\psi}\left(\mathbf{r}_{3}\right)\right| \Phi\right\rangle .
$$

In order to derive a more convenient form of the last expression, we will use a complete orthonormal basis $|\alpha\rangle$, which will be specified later. The states $|\alpha\rangle$ have the properties

$$
\begin{aligned}
\hat{H}|\alpha\rangle & =E_{\alpha}|\alpha\rangle ; \\
\sum_{\alpha}|\alpha\rangle\langle\alpha| & =1, \quad \text { completeness; } \\
\langle\alpha \mid \beta\rangle & =\delta_{\alpha \beta}, \quad \text { orthonormality . }
\end{aligned}
$$

After inserting this completeness relation into (4) we get the following expression for the $p$ - $h$ propagator in the energy representation:

$$
K(\omega)=i \sum_{\alpha}\left\{\frac{\left\langle\Phi\left|\hat{\psi}^{\dagger}\left(\mathbf{r}_{4}\right) \hat{\psi}\left(\mathbf{r}_{3}\right)\right| \alpha\right\rangle\left\langle\alpha\left|\hat{\psi}^{\dagger}\left(\mathbf{r}_{2}\right) \hat{\psi}\left(\mathbf{r}_{1}\right)\right| \Phi\right\rangle}{\omega+E_{\alpha}-E_{0}-i \delta}-\frac{\left\langle\Phi\left|\hat{\psi}^{\dagger}\left(\mathbf{r}_{2}\right) \hat{\psi}\left(\mathbf{r}_{1}\right)\right| \alpha\right\rangle\left\langle\alpha\left|\hat{\psi}^{\dagger}\left(\mathbf{r}_{4}\right) \hat{\psi}\left(\mathbf{r}_{3}\right)\right| \Phi\right\rangle}{\omega-E_{\alpha}+E_{0}+i \delta}\right\}
$$

where $E_{\alpha}$ is the energy of the state $|\alpha\rangle$. The quantities in the numerators are $p-h$ amplitudes which are of the same type as those encountered in the Bethe-Salpeter theory [9]. We denote the excitation energies by $E_{\alpha 0}=E_{\alpha}-E_{0}$ and introduce the spectral function

$$
\begin{aligned}
A\left(\mathbf{r}_{1}, \mathbf{r}_{2}, \mathbf{r}_{3}, \mathbf{r}_{4} ; E\right)=\sum_{\alpha} & {\left[\left\langle\Phi\left|\hat{\psi}^{\dagger}\left(\mathbf{r}_{2}\right) \hat{\psi}\left(\mathbf{r}_{1}\right)\right| \alpha\right\rangle\right.} \\
& \left.\times\left\langle\alpha\left|\hat{\psi}^{\dagger}\left(\mathbf{r}_{4}\right) \hat{\psi}\left(\mathbf{r}_{3}\right)\right| \Phi\right\rangle\right] \delta\left(E-E_{\alpha 0}\right),
\end{aligned}
$$

which finally yields

$$
\begin{aligned}
& K\left(\mathbf{r}_{1}, \mathbf{r}_{2}, \mathbf{r}_{3}, \mathbf{r}_{4} ; \omega\right)=i \int_{0}^{\infty} d E\left\{\frac{A\left(\mathbf{r}_{3}, \mathbf{r}_{4}, \mathbf{r}_{1}, \mathbf{r}_{2} ; E\right)}{\omega+E-i \delta}\right. \\
& \left.-\frac{A\left(\mathbf{r}_{1}, \mathbf{r}_{2}, \mathbf{r}_{3}, \mathbf{r}_{4} ; E\right)}{\omega-E+i \delta}\right\}
\end{aligned}
$$

From this representation it becomes evident that the poles of the $p$ - $h$ propagator $K$ provide the excitation spectrum of the system. Since we are interested in the excitation modes of the Dirac vacuum we will be concerned with an analysis of the poles of the Green's func- tion $K(\omega)$. As we mentioned before, this function is yet unspecified. In order to derive an expression more suitable for our purposes, we will proceed in two steps. In the first step we will construct the corresponding Green's function in the external field without the $p$ - $h$ interaction and in a second step we will use the Bethe-Salpeter equation in a certain approximation in order to construct the propagator incorporating interaction from the free one.

The free Green's function $K_{0}$ has of course the same form as $K$ from Eq. (1), except for the fact that the expectation value of the time-ordered product has to be taken with respect to the noninteracting ground state $\Phi_{0}$, i.e., the free Dirac vacuum. We expand the field operators $\hat{\psi}$ and $\hat{\psi}^{\dagger}$ in a complete orthonormal single-particle basis,

$$
\begin{gathered}
\hat{\psi}(\mathbf{r})=\sum_{p>F} \hat{a}_{p} \varphi_{p}(\mathbf{r})+\sum_{h \leq F} \hat{b}_{h}^{\dagger} \Phi_{h}(\mathbf{r}), \\
\hat{\psi}^{\dagger}(\mathbf{r})=\sum_{p>F} \hat{a}_{p}^{\dagger} \varphi_{p}^{\dagger}(\mathbf{r})+\sum_{h \leq F} \hat{b}_{h} \Phi_{h}^{\dagger}(\mathbf{r}),
\end{gathered}
$$

where the first summations comprise all states above the Fermi level (electron states) while the second incorporate all states below $E_{F}=-m$ (positron states). The $\hat{a}_{p}, \hat{a}_{p}^{\dagger}$ 
$\left(\hat{b}_{h}, \hat{b}_{h}^{\dagger}\right)$ are single-electron (positron) annihilation and creation operators, respectively, satisfying the standard anticommutator relations for fermions. The summation indices $p$ and $h$ denote the quantum numbers of the different states. In a spherically symmetric basis, we have to deal with a radial quantum number $n$, a total angular momentum quantum number $j$, a magnetic quantum number $\mu$, and parity. The $p$ - $h$ states $|\alpha\rangle$ are provided by the action of the introduced single-particle operators on the Dirac vacuum,

$$
|\alpha\rangle=\hat{a}_{p}^{\dagger} \hat{b}_{h}^{\dagger}\left|\Phi_{0}\right\rangle
$$

We make use of the action of the $p-h$ operators on the free ground state:

$$
\begin{aligned}
& \hat{a}_{p}\left|\Phi_{0}\right\rangle=\left\langle\Phi_{0}\right| \hat{a}_{p}^{\dagger}=0, \\
& \hat{b}_{h}\left|\Phi_{0}\right\rangle=\left\langle\Phi_{0}\right| \hat{b}_{h}^{\dagger}=0,
\end{aligned}
$$

which are a direct consequence of the fact that the vacuum contains no particles above the Fermi level and no holes below it. With these prerequisites we obtain the following result for the free Bethe-Salpeter amplitudes:

$$
\begin{aligned}
\left\langle\Phi_{0}\left|\hat{\psi}^{\dagger}\left(\mathbf{r}_{2}\right) \hat{\psi}\left(\mathbf{r}_{1}\right)\right| \alpha\right\rangle & =\left\langle\Phi_{0}\left|\left\{\sum_{p_{2}, h_{1}} \hat{b}_{h_{1}} \hat{a}_{p_{2}}\left[\Phi_{h_{1}}^{\dagger}\left(\mathbf{r}_{2}\right) \varphi_{p_{2}}\left(\mathbf{r}_{1}\right)\right]+\sum_{p_{1}, h_{2}}(\cdots)+\sum_{p_{1}, p_{2}}(\cdots)+\sum_{h_{1}, h_{2}}(\cdots)\right\} \hat{a}_{p}^{\dagger} \hat{b}_{h}^{\dagger}\right| \Phi_{0}\right\rangle \\
& =\Phi_{h}^{\dagger}\left(\mathbf{r}_{2}\right) \varphi_{p}\left(\mathbf{r}_{1}\right)
\end{aligned}
$$

where the dots in this amplitude indicate terms which vanish as a consequence of the fermionic anticommutator relations and Eqs. (11).

After inserting these results in Eq. (6) and replacing the interacting vacuum $\Phi$ by the free one $\Phi_{0}$, we can represent the external field propagator without the $p-h$ interaction by

$$
K_{0}(\omega)=i \sum_{p, h}\left\{\frac{\Phi_{h}^{\dagger}\left(\mathbf{r}_{4}\right) \varphi_{p}\left(\mathbf{r}_{3}\right) \varphi_{p}^{\dagger}\left(\mathbf{r}_{2}\right) \Phi_{h}\left(\mathbf{r}_{1}\right)}{\omega+E_{p}-E_{h}-i \delta}-\frac{\Phi_{h}^{\dagger}\left(\mathbf{r}_{2}\right) \varphi_{p}\left(\mathbf{r}_{1}\right) \varphi_{p}^{\dagger}\left(\mathbf{r}_{4}\right) \Phi_{h}\left(\mathbf{r}_{3}\right)}{\omega-E_{p}+E_{h}+i \delta}\right\},
$$

where $\varphi_{p}\left(\Phi_{h}\right)$ are single-electron (positron) states in the external nuclear Coulomb field with eigenenergies $E_{p}$ and $E_{h}$, respectively.

As the next step we will consider the Bethe-Salpeter equation for the Green's function,

$$
\begin{aligned}
K\left(x_{1}, x_{2}, x_{3}, x_{4}\right)=K_{0}\left(x_{1}, x_{2}, x_{3}, x_{4}\right)+i \int & d^{4} x_{5} d^{4} x_{6} d^{4} x_{7} d^{4} x_{8} K_{0}\left(x_{1}, x_{2}, x_{5}, x_{6}\right) \\
& \times V\left(x_{5}, x_{6}, x_{7}, x_{8}\right) K\left(x_{7}, x_{8}, x_{3}, x_{4}\right)
\end{aligned}
$$

or, if one regards the functions $K, K_{0}$, and $V$ as matrices depending on the continuous space-time variables, one may write in a symbolic notation (Fig. 1)

$$
K(\omega)=K_{0}(\omega)+i K_{0}(\omega) V(\omega) K(\omega) .
$$

This equation is still exact and iterates the interaction kernel $V$ which contains all irreducible self-energy contributions at infinite order. However, here we are faced with a major problem, since the Bethe-Salpeter equation with the inclusion of the full interaction kernel cannot be solved in practice. At this point we introduce the approximation by retaining only the first-order perturbative contribution to $V$, i.e., by considering only one-photon exchange between the particle and the hole. In this special case the kernel $V$ becomes energy independent. This
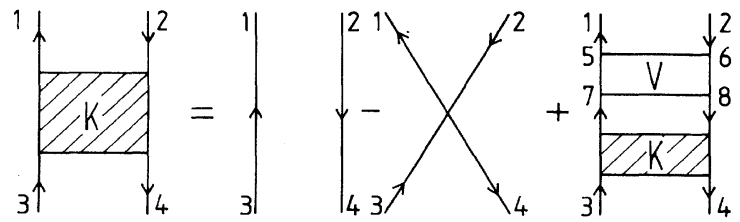

FIG. 1. Diagrammatic representation of the BetheSalpeter equation. is known as the instantaneous ladder approximation in the Bethe-Salpeter theory [9]. In addition to the direct interaction we will take into account the exchange interaction. Finally the kernel $V$ reads

$$
\begin{aligned}
V\left(x_{5}, x_{6}, x_{7}, x_{8}\right)= & v\left(\mathbf{r}_{5}, \mathbf{r}_{6}\right) \delta\left(t_{5}-t_{6}\right) \delta^{(4)}\left(x_{5}-x_{7}\right) \\
& \times \delta^{(4)}\left(x_{6}-x_{8}\right)\left[1-P_{5 \leftrightarrow 6}\right],
\end{aligned}
$$

where $v$ denotes the Coulomb interaction, while $P_{5 \leftrightarrow 6}$ signifies a permutation operator which provides the exchange interaction. The remaining graphs which are iterated by Eq. (14) are illustrated in Fig. 2.

We would like to mention that the inclusion of mag-

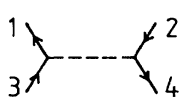

(a)

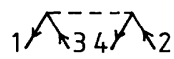

(c)

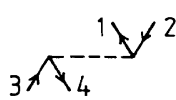

(b)

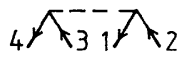

(d)
FIG. 2. First-order irreducible vertex parts. The dashed line indicates the Coulomb interaction. 


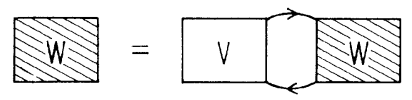

FIG. 3. Diagrammatic representation of the homogeneous equation for $W(\omega)$.

netic and retardation effects as described by the Breit interaction [10] in the iterative expansion would lead to erroneous results, since the contributions of the Breit interaction to the energy of the system have to be taken into account only as a first-order perturbation [11]. This traces back to the fact that the Breit interaction was constructed from the classical Darwin Lagrangian, which takes into account retardation effects by analogy arguments, and is not rigorously derived from quantum field theory.

Returning to the bare $p$ - $h$ interaction $V$ described through the graphs in Fig. 2, it will become clear later that their iterative expansion with the aid of Eq. (14) is identical with the so-called random-phase approximation (RPA), which follows from the time-dependent HartreeFock equations in many-body theory [12].

It is useful to write explicitly the iterative expansion of Eq. (15) with the kernel $V$ replaced by expression (16):

$$
\begin{aligned}
K(\omega)= & K_{0}(\omega)+i K_{0}(\omega)\left\{V+\left[i V K_{0}(\omega)\right] V\right. \\
& \left.+\left[i V K_{0}(\omega)\right]^{2} V+\cdots\right\} K_{0}(\omega) \\
= & K_{0}(\omega)+i K_{0}(\omega) W(\omega) K_{0}(\omega),
\end{aligned}
$$

where we introduced an energy-dependent effective interaction $W(\omega)[6]$, defined by the infinite sum in the curly brackets, i.e.,

$$
W(\omega)=V+i V K_{0}(\omega) W(\omega) .
$$

This last equation for the effective $p$ - $h$ interaction represents the vertex iteration encountered in quantum field theory [13]. We are aiming at a precise determination of the excitation energies of the system. These are just the poles of the propagator $K(\omega)$ including the interaction [see Eq. (8) and the discussion below]. From Eq. (17) it is obvious that the poles of the function $K$ are provided by the known poles of $K_{0}$ which are located at the positions of the unperturbed $p$ - $h$ energies and by the poles of the introduced energy-dependent interaction $W(\omega)$, which are

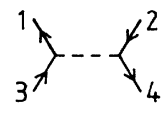

(a)

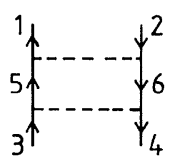

(d)

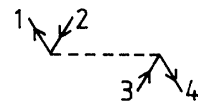

(b)

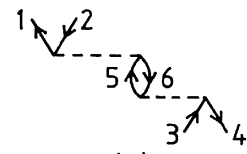

(c)

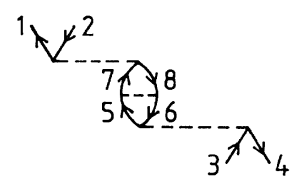

(e)

FIG. 4. Lowest-order contributions to the effective interaction $W(\omega)$.

a direct consequence of the $p$ - $h$ interaction. A brief inspection of Eq. (18) for $W(\omega)$ manifests that the poles of $K_{0}$ correspond to roots of $W(\omega)$, since the formal solution of this equation is simply $W(\omega)=V /\left[1-i V K_{0}(\omega)\right]$. Thus, the advantage provided by the introduction of the energy-dependent effective interaction $W(\omega)$ becomes evident, since Eq. (18) has no poles located at the positions of the unperturbed $p$ - $h$ energies. Consequently, for the determination of excitation energies in which we are interested, we will investigate Eq. (18) for $W(\omega)$. To be more precise, we will be concerned with the homogeneous form of this equation, since in the vicinity of a pole of $W(\omega)$ one can ignore the inhomogeneity $V$, which is energy independent. The resulting homogeneous equation is illustrated diagrammatically in Fig. 3. In addition, the lowest-order contributions to the effective interaction which are iterated by Eq. (18) are depicted in Fig. 4.

\section{REDUCTION OF THE EQUATION FOR THE EFFECTIVE INTERACTION IN COORDINATE REPRESENTATION}

In this section we will deal with the analytical reduction of the homogeneous Bethe-Salpeter equation (18) for the effective $p-h$ interaction. To accomplish this, first it is neccessary to study the coordinate representation of this equation. After inserting the coordinate representation of the external field propagator $K_{0}$ from Eq. (13) we get

$$
\begin{array}{r}
W\left(\mathbf{r}_{1}, \mathbf{r}_{2} ; \omega\right)=\int d^{3} r_{3} \int d^{3} r_{4}\left\{V ( \mathbf { r } _ { 1 } , \mathbf { r } _ { 3 } ) \sum _ { p , h } \left[\frac{\Phi_{h}^{\dagger}\left(\mathbf{r}_{3}\right) \varphi_{p}\left(\mathbf{r}_{3}\right) \varphi_{p}^{\dagger}\left(\mathbf{r}_{4}\right) \Phi_{h}\left(\mathbf{r}_{4}\right)}{\omega-E_{p}+E_{h}+i \delta}\right.\right. \\
\left.\left.-\frac{\Phi_{h}^{\dagger}\left(\mathbf{r}_{4}\right) \varphi_{p}\left(\mathbf{r}_{4}\right) \varphi_{p}^{\dagger}\left(\mathbf{r}_{3}\right) \Phi_{h}\left(\mathbf{r}_{3}\right)}{\omega+E_{p}-E_{h}-i \delta}\right] W\left(\mathbf{r}_{4}, \mathbf{r}_{2} ; \omega\right)\right\},
\end{array}
$$

where $V\left(\mathbf{r}_{1}, \mathbf{r}_{3}\right)$ is the instantaneous Coulomb interaction including exchange, while $\varphi_{p}$ and $\Phi_{h}$ are particle (electron) and hole (positron) Dirac spinors, as provided by the single-particle Dirac equation incorporating the nuclear Coulomb potential. Of course, it is a complicated task to determine directly the solutions of the homogeneous integral equation (19) in six dimensions. Therefore we first project the above equation into the $p$ - $h$ space and consider the various matrix elements. In the resulting equation for the associated matrix elements, which will be investigated in spherical coordinates, one can perform all angular integrations analytically, getting thus an equation in the two radial coordinates. This final equation will then be analyzed numerically in Sec. IV.

First, we define the occupation number $n_{\alpha}$ according to 


$$
n_{\alpha}=\left\{\begin{array}{lr}
1, & E_{\alpha} \leq E_{F} \text { (holes) } \\
0, & E_{\alpha}>E_{F} \quad \text { (particles) }
\end{array}\right.
$$

which we insert in Eq. (18). This yields

$$
\begin{gathered}
W\left(\mathbf{r}_{1}, \mathbf{r}_{2} ; \omega\right)=-\int d^{3} r_{3} \int d^{3} r_{4}\left\{\sum_{\alpha_{5}, \alpha_{6}}\left(n_{\alpha_{6}}-n_{\alpha_{5}}\right)\left[\psi_{\alpha_{5}}^{\dagger}\left(\mathbf{r}_{3}\right) \psi_{\alpha_{6}}^{\dagger}\left(\mathbf{r}_{4}\right) \frac{V\left(\mathbf{r}_{1}, \mathbf{r}_{3}\right)}{\omega-E_{\alpha_{6}}+E_{\alpha_{5}}+i \delta\left(n_{\alpha_{5}}-n_{\alpha_{6}}\right)} \psi_{\alpha_{5}}\left(\mathbf{r}_{4}\right) \psi_{\alpha_{6}}\left(\mathbf{r}_{3}\right)\right]\right. \\
\left.\times W\left(\mathbf{r}_{4}, \mathbf{r}_{2} ; \omega\right)\right\}
\end{gathered}
$$

where the summation indices $\alpha_{5}$ and $\alpha_{6}$ run over all single-particle states, which are designated by the external field Dirac spinors $\psi$. The above equation possesses nontrivial solutions only if the state $\alpha_{6}$ contains a particle and the state $\alpha_{5}$ a hole, or vice versa, since this equation describes $p$ - $h$ propagation by construction. Next we project Eq. (21) into the $p$ - $h$ channel by defining the following matrix elements:

$$
\left\langle\alpha_{1} \alpha_{3}|W| \alpha_{2} \alpha_{4}\right\rangle=\int d^{3} r_{1} \int d^{3} r_{2} \psi_{\alpha_{1}}^{\dagger}\left(\mathbf{r}_{1}\right) \psi_{\alpha_{3}}^{\dagger}\left(\mathbf{r}_{2}\right) W\left(\mathbf{r}_{1}, \mathbf{r}_{2}\right) \psi_{\alpha_{2}}\left(\mathbf{r}_{1}\right) \psi_{\alpha_{4}}\left(\mathbf{r}_{2}\right)
$$

Finally we derive the following equation of the effective interaction, which is equivalent to the original Eq. (21):

$$
\left\langle\alpha_{1} \alpha_{3}|W| \alpha_{2} \alpha_{4}\right\rangle=\sum_{\alpha_{5}, \alpha_{6}}\left(n_{\alpha_{6}}-n_{\alpha_{5}}\right) \frac{\left\langle\alpha_{1} \alpha_{6}|V| \alpha_{2} \alpha_{5}\right\rangle\left\langle\alpha_{5} \alpha_{3}|W| \alpha_{6} \alpha_{4}\right\rangle}{\omega-E_{\alpha_{5}}+E_{\alpha_{6}}-i \delta\left(n_{\alpha_{5}}-n_{\alpha_{6}}\right)}
$$

Here the matrix element of the bare interaction $V$ is understood to be antisymmetrized, thus taking into account exchange effects,

$$
\left\langle\alpha_{1} \alpha_{6}|V| \alpha_{2} \alpha_{5}\right\rangle=\left\langle\alpha_{1} \alpha_{6}|v| \alpha_{2} \alpha_{5}\right\rangle-\left\langle\alpha_{1} \alpha_{6}|v| \alpha_{5} \alpha_{2}\right\rangle
$$

where $v\left(\mathbf{r}_{1}, \mathbf{r}_{2}\right)=\alpha /\left|\mathbf{r}_{1}-\mathbf{r}_{2}\right|$ is the instantaneous Coulomb interaction, while $\alpha=e^{2} /(4 \pi) \simeq 1 / 137$ is the fine-structure constant. Now, we have to specify explicitly the single-particle states $\left\langle\mathbf{r} \mid \alpha_{i}\right\rangle=\psi_{\alpha_{i}}(\mathbf{r})$, in order to implement the symmetries and conservation laws. The external field is assumed to possess spherical symmetry and the functions $\psi_{\alpha}(\mathbf{r})$ are eigensolutions of the Dirac equation for the nuclear Coulomb potential. The nuclear charge distribution is assumed to be a homogeneously charged sphere of radius $R$, i.e.,

$$
\left\{\boldsymbol{\alpha} \cdot \mathbf{p}+\beta m+V_{\text {nuc }}(r)\right\} \psi_{\alpha}(\mathbf{r})=E_{\alpha} \psi_{\alpha}(\mathbf{r}),
$$

with

$$
V_{\text {nuc }}(r)=\left\{\begin{array}{l}
-\frac{Z \alpha}{2 R}\left[3-\left(\frac{r}{R}\right)^{2}\right], \quad r \leq R \\
-\frac{Z \alpha}{r}, \quad r>R
\end{array}\right.
$$

Here we used the parametrization $R=r_{0} A^{1 / 3}, r_{0}=1.2$ $\mathrm{fm}$, and $A=2.5 Z$, the relation between the mass number $A$ and the charge number $Z$ being valid for the heavy nuclei considered in this paper. In a spherically symmetric external field the Dirac spinors $\psi_{\alpha}$ are simultaneously eigensolutions of the energy operator $\hat{H}$, the total angular momentum operator $\hat{J}^{2}$, its projection on the $z$ axis $\hat{J}_{z}$, and the operator $\hat{K}=\beta(\boldsymbol{\sigma} \cdot \mathbf{L}+1)$ with the eigenvalues $E_{\alpha}, j, \mu,-\kappa$, respectively [14]. For the wave functions $\psi_{\alpha}$ we make the usual spherical ansatz

$$
\psi_{\alpha}(\mathbf{r})=\left(\begin{array}{c}
g_{\kappa}(r) \chi_{\kappa \mu}(\Omega) \\
i f_{\kappa}(r) \chi-\kappa \mu \\
(\Omega)
\end{array}\right),
$$

where $g_{\kappa}(r)$ and $f_{\kappa}(r)$ are radial functions, while $\chi_{\kappa \mu}(\Omega)$ are two-component spherical spinors defined as [14]

$$
\chi_{\kappa \mu}(\Omega)=(-1)^{1 / 2-l-\mu} \sum_{m= \pm 1 / 2} \sqrt{2 j+1}\left(\begin{array}{ccc}
l & \frac{1}{2} & j \\
\mu-m & m & -\mu
\end{array}\right) Y_{l, \mu-m}(\Omega) \tilde{\chi}_{1 / 2, m}
$$

where $l$ is the orbital momentum quantum number, $Y_{l, \mu-m}(\Omega)$ are spherical harmonics, and the $\tilde{\chi}_{1 / 2, m}$ are twocomponent Pauli spinors, respectively. The index $\alpha$ in the ansatz (27) denotes the set of quantum numbers $n, \kappa$, and $\mu$. Next, we introduce the multipole decomposition of the Coulomb interaction [15]

$$
v\left(\mathbf{r}_{1}, \mathbf{r}_{2}\right)=\alpha \sum_{L=0}^{\infty} \sum_{M=-L}^{L} \frac{4 \pi}{2 L+1} \frac{r_{<}^{L}}{r_{>}^{L+1}} Y_{L, M}^{*}\left(\Omega_{2}\right) Y_{L, M}\left(\Omega_{1}\right)
$$

where $r_{<}=\min \left(r_{1}, r_{2}\right)$, etc. With these preliminaries the direct interaction $\mathcal{D}$ can be written as 


$$
\begin{aligned}
\mathcal{D}=\left\langle\alpha_{1} \alpha_{3}|v| \alpha_{2} \alpha_{4}\right\rangle= & \int d^{3} r_{1} \int d^{3} r_{2} \psi_{\alpha_{1}}^{\dagger}\left(\mathbf{r}_{1}\right) \psi_{\alpha_{3}}^{\dagger}\left(\mathbf{r}_{2}\right) \frac{\alpha}{\left|\mathbf{r}_{1}-\mathbf{r}_{2}\right|} \psi_{\alpha_{2}}\left(\mathbf{r}_{1}\right) \psi_{\alpha_{4}}\left(\mathbf{r}_{2}\right) \\
=\sum_{L, M}\left(\int_{0}^{\infty} d r_{1} \int_{0}^{\infty} d r_{2}\left(r_{1} r_{2}\right)^{2} \frac{4 \pi}{2 L+1} \frac{r_{L}^{L}}{r_{>}^{L+1}}\right. & \\
\times & \left\{\left[g_{\kappa_{1}}\left(r_{1}\right) g_{\kappa_{2}}\left(r_{1}\right)\right]\left[g_{\kappa_{3}}\left(r_{2}\right) g_{\kappa_{4}}\left(r_{2}\right)\right] I_{g g}+\left[g_{\kappa_{1}}\left(r_{1}\right) g_{\kappa_{2}}\left(r_{1}\right)\right]\left[f_{\kappa_{3}}\left(r_{2}\right) f_{\kappa_{4}}\left(r_{2}\right)\right] I_{g f}\right. \\
+ & {\left.\left.\left[f_{\kappa_{1}}\left(r_{1}\right) f_{\kappa_{2}}\left(r_{1}\right)\right]\left[f_{\kappa_{3}}\left(r_{2}\right) f_{\kappa_{4}}\left(r_{2}\right)\right] I_{f f}+\left[f_{\kappa_{1}}\left(r_{1}\right) f_{\kappa_{2}}\left(r_{1}\right)\right]\left[g_{\kappa_{3}}\left(r_{2}\right) g_{\kappa_{4}}\left(r_{2}\right)\right] I_{f g}\right\}\right), }
\end{aligned}
$$

where we introduced the abbreviations

$I_{g g}=\left\langle\kappa_{1} \mu_{1}\left|Y_{L M}\right| \kappa_{2} \mu_{2}\right\rangle\left\langle\kappa_{3} \mu_{3}\left|Y_{L M}^{*}\right| \kappa_{4} \mu_{4}\right\rangle$,

$I_{g f}=\left\langle\kappa_{1} \mu_{1}\left|Y_{L M}\right| \kappa_{2} \mu_{2}\right\rangle\left\langle-\kappa_{3} \mu_{3}\left|Y_{L M}^{*}\right|-\kappa_{4} \mu_{4}\right\rangle$,

$I_{f f}=\left\langle-\kappa_{1} \mu_{1}\left|Y_{L M}\right|-\kappa_{2} \mu_{2}\right\rangle\left\langle-\kappa_{3} \mu_{3}\left|Y_{L M}^{*}\right|-\kappa_{4} \mu_{4}\right\rangle$,

$I_{f g}=\left\langle-\kappa_{1} \mu_{1}\left|Y_{L M}\right|-\kappa_{2} \mu_{2}\right\rangle\left\langle\kappa_{3} \mu_{3}\left|Y_{L M}^{*}\right| \kappa_{4} \mu_{4}\right\rangle$.

The matrix elements of the spherical harmonics are defined as $(d \Omega=\sin \vartheta d \vartheta d \varphi ;-\pi / 2 \leq \vartheta \leq \pi / 2,0 \leq \varphi \leq 2 \pi)$

$$
\left\langle\kappa_{i} \mu_{i}\left|Y_{L M}\right| \kappa_{j} \mu_{j}\right\rangle=\int d \Omega \chi_{\kappa_{i} \mu_{i}}^{\dagger}(\Omega) Y_{L M}(\Omega) \chi_{\kappa_{j} \mu_{j}}(\Omega) .
$$

These angular integrals can be evaluated by means of the Wigner-Eckhart theorem. As an example we consider

$$
\begin{aligned}
\left\langle\kappa_{1} \mu_{1}\left|Y_{L M}\right| \kappa_{2} \mu_{2}\right\rangle= & (-1)^{j_{1}-\mu_{1}}\left(\begin{array}{ccc}
j_{2} & j_{1} & L \\
\mu_{2} & -\mu_{1} & M
\end{array}\right) \\
& \times\left(\kappa_{1}\left\|Y_{L}\right\| \kappa_{2}\right) P\left(l_{2}+l_{1}+L\right),
\end{aligned}
$$

where the reduced matrix element on the right-hand side of this expression is $M$ independent:

$$
\begin{aligned}
\left(\kappa_{1}\left\|Y_{L}\right\| \kappa_{2}\right)= & (-1)^{\kappa_{1}} \sqrt{\frac{\left|\kappa_{1} \kappa_{2}\right|(2 L+1)}{\pi}} \\
& \times\left(\begin{array}{ccc}
j_{2} & j_{1} & L \\
-\frac{1}{2} & \frac{1}{2} & 0
\end{array}\right)^{\pi} .
\end{aligned}
$$

The factor $P\left(l_{2}+l_{1}+L\right)$ in Eq. (33) is equal to 1 or 0 depending on whether $l_{2}+l_{1}+L$ is an even number or not. This is a direct consequence of parity conservation. With these relations we evaluate the angular integrals from Eq. (31) and obtain finally the following result for the direct term in Eq. (30):

$$
\begin{aligned}
\mathcal{D}= & \left\langle\alpha_{1} \alpha_{3}|v| \alpha_{2} \alpha_{4}\right\rangle \\
= & \sum_{L, M}(-1)^{\mu_{1}+\mu_{3}+M+1}\left(\begin{array}{ccc}
j_{1} & L & j_{2} \\
-\mu_{1} & M & \mu_{2}
\end{array}\right) \\
& \quad \times\left(\begin{array}{ccc}
j_{3} & L & j_{4} \\
-\mu_{3} & -M & \mu_{4}
\end{array}\right)\left(\beta_{1} \beta_{3}\left\|v_{L}\right\| \beta_{2} \beta_{4}\right),
\end{aligned}
$$

where $\beta$ denotes the quantum numbers $n$ and $j$. The reduced matrix element in Eq. (35) displays the explicit structure

$$
\begin{aligned}
\left(\beta_{1} \beta_{3}\left\|v_{L}\right\| \beta_{2} \beta_{4}\right)= & 4 \sqrt{\left|\kappa_{1} \kappa_{2} \kappa_{3} \kappa_{4}\right|}\left(\begin{array}{ccc}
j_{1} & L & j_{2} \\
\frac{1}{2} & 0 & -\frac{1}{2}
\end{array}\right)\left(\begin{array}{ccc}
j_{3} & L & j_{4} \\
\frac{1}{2} & 0 & -\frac{1}{2}
\end{array}\right) \\
& \times \int_{0}^{\infty} d r_{1} \int_{0}^{\infty} d r_{2} R_{\kappa_{1}}^{\dagger}\left(r_{1}\right) R_{\kappa_{3}}^{\dagger}\left(r_{2}\right) \frac{r_{<}^{L}}{r_{>}^{L+1}} R_{\kappa_{2}}\left(r_{1}\right) R_{\kappa_{4}}\left(r_{2}\right),
\end{aligned}
$$

where we defined the radial two-component functions $R_{\kappa}(r)$ according to

$$
R_{\kappa}(r)=r\left(\begin{array}{c}
g_{\kappa}(r) \\
i f_{\kappa}(r)
\end{array}\right)
$$

A similar evaluation of the second term from Eq. (24) yields the following antisymmetrized matrix element for the complete $p-h$ interaction which enters into Eq. (23):

$$
\begin{aligned}
\left\langle\alpha_{1} \alpha_{3}|V| \alpha_{2} \alpha_{4}\right\rangle= & \sum_{L, M}(-1)^{\mu_{1}+\mu_{3}+M+1}\left(\begin{array}{ccc}
j_{1} & L & j_{2} \\
-\mu_{1} & M & \mu_{2}
\end{array}\right)\left(\begin{array}{ccc}
j_{3} & L & j_{4} \\
-\mu_{3} & -M & \mu_{4}
\end{array}\right)\left(\beta_{1} \beta_{3}\left\|v_{L}\right\| \beta_{2} \beta_{4}\right) \\
& -\sum_{L^{\prime}, M^{\prime}}(-1)^{\mu_{1}+\mu_{3}+M^{\prime}+1}\left(\begin{array}{ccc}
j_{1} & L^{\prime} & j_{4} \\
-\mu_{1} & M^{\prime} & \mu_{4}
\end{array}\right)\left(\begin{array}{ccc}
j_{3} & L^{\prime} & j_{2} \\
-\mu_{3} & -M^{\prime} & \mu_{2}
\end{array}\right)\left(\beta_{1} \beta_{3}\left\|v_{L^{\prime}}\right\| \beta_{4} \beta_{2}\right) .
\end{aligned}
$$

In order to obtain a more compact form for this last expression we define reduced matrix elements for the complete bare interaction according to 


$$
\left\langle\alpha_{1} \alpha_{3}|V| \alpha_{2} \alpha_{4}\right\rangle=\sum_{L, M}(-1)^{\mu_{1}+\mu_{3}+M+1}\left(\begin{array}{lll}
j_{1} & L & j_{2} \\
-\mu_{1} & M & \mu_{2}
\end{array}\right)\left(\begin{array}{ccc}
j_{3} & L & j_{4} \\
-\mu_{3} & -M & \mu_{4}
\end{array}\right)\left(\beta_{1} \beta_{3}\left\|V_{L}\right\| \beta_{2} \beta_{4}\right)
$$

By comparing Eqs. (39) and (38) we obtain the following relation between the reduced matrix elements of $V_{L}$ and $v_{L}$ :

$$
\left(\beta_{1} \beta_{3}\left\|V_{L}\right\| \beta_{2} \beta_{4}\right)=\left(\beta_{1} \beta_{3}\left\|v_{L}\right\| \beta_{2} \beta_{4}\right)-\sum_{L^{\prime}}(-1)^{L-L^{\prime}-1}(2 L+1)\left\{\begin{array}{ccc}
j_{1} & L & j_{2} \\
j_{3} & L^{\prime} & j_{4}
\end{array}\right\}\left(\beta_{1} \beta_{3}\left\|v_{L^{\prime}}\right\| \beta_{4} \beta_{2}\right) .
$$

The term which contains the infinite sum over the angular momentum $L^{\prime}$ represents the exchange interaction. In the remaining part of this section we will implement these results into Eq. (23), which will provide an equation for the reduced matrix elements of the effective interaction $W$. We define these matrix elements in analogy to Eq. (39):

$$
\left\langle\alpha_{1} \alpha_{3}|W| \alpha_{2} \alpha_{4}\right\rangle=\sum_{L, M}(-1)^{\mu_{1}+\mu_{3}+M+1}\left(\begin{array}{lll}
j_{1} & L & j_{2} \\
-\mu_{1} & M & \mu_{2}
\end{array}\right)\left(\begin{array}{ccc}
j_{3} & L & j_{4} \\
-\mu_{3} & -M & \mu_{4}
\end{array}\right)\left(\beta_{1} \beta_{3}\left\|W_{L}\right\| \beta_{2} \beta_{4}\right)
$$

After inserting the multipole decompositions (41) and (39) into the original Eq. (23) one obtains after a lengthy but straightforward calculation the following equation for the reduced matrix elements of the effective interaction $W$ :

$$
\left(\beta_{1} \beta_{3}\left\|W_{L}\right\| \beta_{2} \beta_{4}\right)=\sum_{\beta_{5}, \beta_{6}}\left(n_{\beta_{6}}-n_{\beta_{5}}\right) \frac{\left(\beta_{1} \beta_{6}\left\|V_{L}\right\| \beta_{2} \beta_{5}\right)\left(\beta_{5} \beta_{3}\left\|W_{L}\right\| \beta_{6} \beta_{4}\right)}{(2 L+1)\left[\omega-E_{\beta_{5}}+E_{\beta_{6}}-i \delta\left(n_{\beta_{5}}-n_{\beta_{6}}\right)\right]}
$$

The reduced matrix elements of the bare interaction $V_{L}$ are presented in Eqs. (36) and (40). We would like to note that the above equation is valid independently for each value of the angular momentum $L$.

As we argued in Sec. II, the physical content of our approach is completely equivalent to the relativistic random-phase approximation, which already has been extensively employed in the investigation of many-particle systems. To verify this, we consider the original equation (23) for the matrix elements of $W$. Since the summation on the right-hand side of this equation describes the situation in which $\alpha_{5}$ is a hole and $\alpha_{6}$ a particle and vice versa, there are actually two independent summations which are contained in this term. In the following we label explicitly the particle states by $n, m$ and the hole states by $i, j$. An inspection of Eq. (23) shows that there are two coupled equations, depending on whether the index $\alpha$ describes a particle $(\alpha=m, n, \ldots)$ or a hole $(\alpha=i, j, \ldots)$ state, respectively. It is instructive to consider these two equations explicitly:

(i) $\alpha_{1}=m$ (particle) , $\alpha_{2}=i$ (hole):

$$
\begin{aligned}
\left\langle m \alpha_{3}|W| i \alpha_{4}\right\rangle=\sum_{n, j}\{ & \frac{\langle m j|V| i n\rangle\left\langle n \alpha_{3}|W| j \alpha_{4}\right\rangle}{\omega-E_{n}+E_{j}+i \delta} \\
& \left.-\frac{\langle m n|V| i j\rangle\left\langle j \alpha_{3}|W| n \alpha_{4}\right\rangle}{\omega-E_{j}+E_{n}-i \delta}\right\} ;
\end{aligned}
$$

(ii) $\alpha_{1}=i$ (hole),$\alpha_{2}=m$ (particle):

$$
\begin{aligned}
\left\langle i \alpha_{3}|W| m \alpha_{4}\right\rangle=\sum_{n, j}\{ & \frac{\langle i j|V| m n\rangle\left\langle n \alpha_{3}|W| j \alpha_{4}\right\rangle}{\omega-E_{n}+E_{j}+i \delta} \\
& \left.-\frac{\langle i n|V| m j\rangle\left\langle j \alpha_{3}|W| n \alpha_{4}\right\rangle}{\omega-E_{j}+E_{n}-i \delta}\right\} .
\end{aligned}
$$

We emphasize the explicit appearance of the different types of couplings contained in the above equations, i.e. $p-p, p-h$, and $h-h$ interactions. Next, we perform the following substitutions:

$$
\begin{aligned}
& \frac{\left\langle\alpha_{3} n|W| \alpha_{4} j\right\rangle}{\omega-E_{n}+E_{j}+i \delta}=x_{n j}(\omega), \\
& \frac{\left\langle\alpha_{3} j|W| \alpha_{4} n\right\rangle}{\omega-E_{j}+E_{n}-i \delta}=-y_{n j}(\omega),
\end{aligned}
$$

which we insert into the set of Eqs. (43) and (44). This leads to

$$
\begin{aligned}
\left(\omega-E_{m}+E_{i}\right) x_{m i}=\sum_{n, j}\{ & \langle m j|V| i n\rangle x_{n j} \\
& \left.+\langle m n|V| i j\rangle y_{n j}\right\}, \\
-\left(\omega-E_{i}+E_{m}\right) y_{m i}=\sum_{n, j}\{ & \langle i j|V| m n\rangle x_{n j} \\
& \left.+\langle i n|V| m j\rangle y_{n j}\right\},
\end{aligned}
$$

where we employed the symmetry $\langle 12|V| 34\rangle=\langle 21|V| 43\rangle$ etc., of the matrix elements of the Coulomb interaction. The two sets of coupled equations (46) are exactly the eigenvalue equations of the random-phase approximation for the energy-dependent RPA amplitudes $x_{n j}$ and $y_{n j}$ [16]. These amplitudes describe physically the transition probability of particle-hole pairs between excited RPA states $\left|\psi_{\mathrm{RPA}}\right\rangle$ and the RPA-correlated vacuum $\left|0_{\mathrm{RPA}}\right\rangle$. We would like to mention that the Tamm-Dancoff equation [17] follows immediately from Eqs. (46) in the case that these decouple. This happens when the amplitudes $y_{n j}$ can be ignored:

$\sum_{n, j}\left\{\delta_{m n} \delta_{i j}\left(\omega-E_{n}+E_{j}\right)-\langle m j|V| i n\rangle\right\} x_{n j}=0$.

The range of applicability of this equation is determined 
by the condition $\langle V\rangle /\left(\omega-E_{m}+E_{i}\right) \ll 1$, i.e., the expectation value of the bare $p$ - $h$ interaction has to be small compared with the difference between the excitation energies $\omega$ of the various $p-h$ configurations and the unperturbed $p-h$ eigenenergies $E_{p}-E_{h}$. As a consequence of neglecting these so-called backgoing amplitudes $y_{n j}$, the Tamm-Dancoff approximation allows only for one particle and one hole in the intermediate states [Fig. 5(a)]. This feature is different in the RPA, where in the intermediate states any number of particle-hole pairs may be present [Fig. 5(b)].

At the end of this section we study multipole transitions in the spherically symmetric external nuclear field. We will present the general formulas which describe the influence of $p$ - $h$ correlations on the line intensity for the transition of an electron from an initial state $|i\rangle$ to a final state $|f\rangle$. If the corresponding values differ strongly from their bare values without the $p-h$ interaction, one can conclude that correlation effects are strong, and as a consequence, that these correlations may lead to the formation of collective excited states in the considered system.

The interaction of the electron with the quantized radiation field is described by the matrix element [13]

$$
|\langle f|\boldsymbol{\alpha} \cdot \mathbf{A}| i\rangle|^{2}=\left|\left\langle f\left|\boldsymbol{\alpha} \cdot \mathbf{u}_{p} e^{i \mathbf{k} \cdot \mathbf{r}}\right| i\right\rangle\right|^{2},
$$

where $\mathbf{u}_{p}$ is a polarization vector and the vector $\boldsymbol{\alpha}$ designates the Dirac matrices. After expanding the photon field into multipoles, the transition rate per unit time $\Gamma_{i f}$ becomes [18]

$$
\begin{aligned}
\Gamma_{i f}=4 \pi^{2} \alpha \omega \sum_{L, M} & {\left[\left|\left\langle f\left|\boldsymbol{\alpha} \cdot \mathbf{A}_{L M}(m)\right| i\right\rangle\right|^{2}\right.} \\
& \left.+\left|\left\langle f\left|\boldsymbol{\alpha} \cdot \mathbf{A}_{L M}(e)\right| i\right\rangle\right|^{2}\right]
\end{aligned}
$$

with $(\hat{\mathbf{L}}=-i \mathbf{r} \times \boldsymbol{\nabla})$

$\mathbf{A}_{L M}(m)=i \sqrt{2 / \pi} j_{L}(\omega r) \frac{1}{\sqrt{L(L+1)}} \hat{\mathbf{L}} Y_{L M}(\Omega)$,

$$
\mathbf{A}_{L M}(e)=\sqrt{2 / \pi} \omega \frac{1}{\sqrt{L(L+1)}} \nabla \times\left[\hat{\mathbf{L}} j_{L}(\omega r) Y_{L_{M}}(\Omega)\right] .
$$

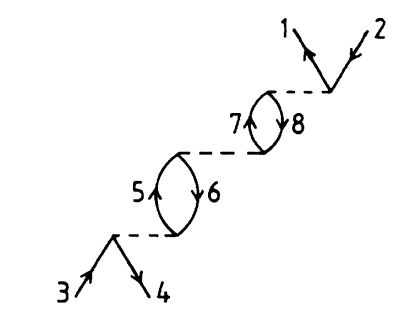

(a)

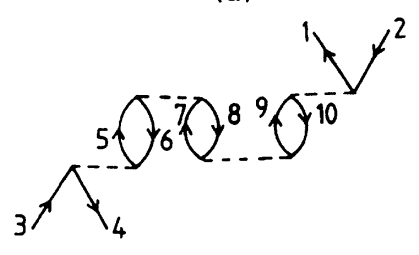

(b)

FIG. 5. Intermediate states in the Tamm-Dancoff approximation (a) and in RPA (b).

After averaging over the magnetic quantum numbers of the final state we find

$$
\Gamma_{i f}=2 \alpha \omega^{2} \sum_{L}\left[f_{L}(m)+f_{L}(e)\right]
$$

with

$$
\begin{gathered}
f_{L}(m)=\frac{2 j_{i}+1}{\omega} B\left(-\kappa_{i}, \kappa_{f}, L\right) M_{L}^{2}, \\
f_{L}(e)=\frac{2 j_{i}+1}{\omega} B\left(\kappa_{i}, \kappa_{f}, L\right) E_{L}^{2},
\end{gathered}
$$

where $j=|\kappa|-\frac{1}{2}$, while the functions $B$ are those given in Ref. [18],

$$
\begin{aligned}
B\left(\kappa_{i}, \kappa_{f}, L\right) & =\frac{\left(2 l_{i}+1\right)\left(2 l_{f}+1\right)}{L(L+1)} C^{2}\left(l_{i}, l_{f}, L ; 0,0\right) W^{2}\left(j_{i} l_{i}, j_{f} l_{f} ; 1 / 2 L\right) \\
C^{2}\left(l_{i}, l_{f}, L ; 0,0\right) & =\frac{(2 L+1)(J-2 L) !\left(J-2 l_{i}\right) !\left(J-2 l_{f}\right) !}{(J+1) !}\left[\frac{(J / 2) !}{(J / 2-L) !\left(J / 2-l_{i}\right) !\left(J / 2-l_{f}\right) !}\right]^{2} \\
W^{2}\left(j_{i} l_{i}, j_{f} l_{f} ; 1 / 2 L\right) & =\left|\frac{\left(L+\kappa_{i}+\kappa_{f}+1\right)\left(\kappa_{i}+\kappa_{f}-L\right)}{4 \kappa_{i} \kappa_{f}\left(2 \kappa_{i}+1\right)\left(2 \kappa_{f}+1\right)}\right|^{2} .
\end{aligned}
$$

We note that the argument $-\kappa_{i}$ in the function $B$ from Eqs. (52) and (53) requires a definition of the orbital momentum quantum number $l$ as $l=\kappa$ for $\kappa>0$ and $l=-\kappa-1$ for $\kappa<0$, respectively. The radial matrix elements are

$$
\begin{aligned}
& M_{L}=\left(\kappa_{i}+\kappa_{f}\right) \int_{0}^{\infty} d r j_{L}(\omega r)\left(F_{f} G_{i}+G_{f} F_{i}\right) \\
& E_{L}=\int_{0}^{\infty} d r\left\{j_{L-1}(\omega r)\left[\left(\kappa_{f}-\kappa_{i}\right)\left(F_{f} G_{i}+G_{f} F_{i}\right)+L\left(F_{f} G_{i}-G_{f} F_{i}\right)\right]+L\left(G_{f} G_{i}+F_{f} F_{i}\right) j_{L}(\omega r)\right\} .
\end{aligned}
$$


$j_{L}(\omega r)$ denotes the spherical Bessel functions. For convenience we considered in our calculations the quantities $f_{L}$ from Eqs. (52) multiplied by the photon energy $\omega$, the advantage being that the resulting quantities are dimensionless. The corresponding quantities with $p$ - $h$ correlations $\bar{f}_{L}$ can be obtained by considering the expansion of a general single-particle operator $\hat{O}$ in terms of the $p$ - $h$ operators [19]

$\left\langle\psi_{0}|\hat{O}| \psi_{n}\right\rangle=\sum_{p, h}\left\{\langle h|\hat{O}| p\rangle x_{p h}^{(n)}+\langle p|\hat{O}| h\rangle y_{p h}^{(n)}\right\}$

where $\left|\psi_{0}\right\rangle$ and $\left|\psi_{n}\right\rangle$ represent the ground and RPA- excited states, respectively, while $x_{p h}^{(n)}$ and $y_{p h}^{(n)}$ are the eigensolutions of Eqs. (46). Employing this last relation, one easily incorporates the $p$ - $h$ interaction into the radial matrix elements (54) which provide the corresponding quantities (52) with the inclusion of correlations.

\section{NUMERICAL ANALYSIS}

First we discuss the solution of Eq. (42) for the reduced matrix elements of the effective $p$ - $h$ interaction $W_{L}$. Some computational details will be also given. It is useful to regard this equation in the following form:

$$
\sum_{\beta_{5}, \beta_{6}}\left\{\delta_{\beta_{1} \beta_{5}} \delta_{\beta_{2} \beta_{6}}+\frac{n_{\beta_{5}}-n_{\beta_{6}}}{2 L+1} \frac{\left(\beta_{1} \beta_{6}\left\|V_{L}\right\| \beta_{2} \beta_{5}\right)}{\omega-E_{\beta_{5}}+E_{\beta_{6}}-i \delta\left(n_{\beta_{5}}-n_{\beta_{6}}\right)}\right\}\left(\beta_{5} \beta_{3}\left\|W_{L}\right\| \beta_{6} \beta_{4}\right)=0
$$

This represents a homogeneous system of algebraic equations which admits solutions only if the determinant of the matrix in the curly brackets is equal to zero. Thus, if we define the matrix $\hat{M}$ with corresponding elements

$$
M_{\left(\beta_{1} \beta_{2}\right)\left(\beta_{3} \beta_{4}\right)}=\delta_{\beta_{1} \beta_{3}} \delta_{\beta_{2} \beta_{4}}+\frac{n_{\beta_{3}}-n_{\beta_{4}}}{2 L+1} \frac{\left(\beta_{1} \beta_{4}\left\|V_{L}\right\| \beta_{2} \beta_{3}\right)}{\omega-E_{\beta_{3}}+E_{\beta_{4}}-i \delta\left(n_{\beta_{3}}-n_{\beta_{4}}\right)},
$$

the condition

$$
\operatorname{det}[\hat{M}(\omega)]=0
$$

determines the eigenvalues $\omega$ for which Eq. (56) exhibits nontrivial solutions. We note that the four indices in Eq. (57) are independent. There is only the restriction that if two of them refer to states above the Fermi level, the other two have to represent states below it. If we denote the number of particle states by $N_{p}$ and the number of hole states by $N_{h}$, the dimension of the matrix $\hat{M}$ is exactly $\left(N_{p} \times N_{h}\right)^{2}$. Since in principle the matrix $\hat{M}$ is infinite dimensional, a truncation has to be introduced. Some aspects of this truncation will be discussed later when we evaluate the line intensities, which are intimately connected with the transition probabilities. The numerical treatment consists of two steps. In a first step we calculated the matrix elements of the bare $p$ - $h$ interaction, which explicitly enters into the matrix $\hat{M}(\omega)$ from Eq. (57), while in a second step we determine the eigenvalues $\omega$ which are the roots of Eq. (58). The states which enter into the matrix elements are solutions of the radial single-particle Dirac equation with the external nuclear potential which follows from Eq. (26):

$\frac{d}{d r}\left(\begin{array}{c}G(r) \\ F(r)\end{array}\right)=\left(\begin{array}{cc}-\kappa / r & E+1-V_{\text {nuc }} \\ -E+1-V_{\text {nuc }} & \kappa / r\end{array}\right)\left(\begin{array}{c}G(r) \\ F(r)\end{array}\right)$.

$G(r)=r g(r)$ and $F(r)=r f(r)$ are the so-called large and small radial wave functions which enter into the spherical ansatz (27), respectively. Depending on whether the single-particle energy $E$ is located in the gap $(-m \leq E \leq m)$, or not $(|E|>m)$, the radial functions exhibit totally different properties. For the investigated superheavy systems, the bound-state solutions are highly localized and decrease rapidly with large values of $r$. The bound-state wave functions are conventionally normalized according to

$$
\int_{V} d^{3} r \psi^{\dagger}(\mathbf{r}) \psi(\mathbf{r})=\int_{0}^{\infty} d r\left(G^{2}+F^{2}\right)=1,
$$

where $\rho(\mathbf{r})=\psi^{\dagger}(\mathbf{r}) \psi(\mathbf{r})$ represents the density. The continuum states are described by oscillatory wave functions with typical sine and cosine behavior in the asymptotic region. In contrast to Eq. (60), these wave functions are normalized on the energy scale, i.e.,

$$
\int_{0}^{\infty} d r\left\{G_{E^{\prime}}(r) G_{E}(r)+F_{E^{\prime}}(r) F_{E}(r)\right\}=\delta\left(E-E^{\prime}\right) .
$$

Convergence problems arise in the evaluation of the radial interaction matrix elements as a consequence of the behavior of continuum wave functions at $r \rightarrow \infty$. In order to circumvent this difficulty, we have discretized the continuum by constructing relativistic wave packets [20]. Relativistic wave packets have been extensively exploited in calculations of electron excitation processes, where it was demonstrated that they are well suited for the description of the continuum. The wave packets are obtained from the radial continuum wave functions after an integration on the energy scale, i.e.,

$$
\begin{aligned}
& u_{1}(r)=\frac{1}{\Delta E} \int_{E_{0}-\Delta E / 2}^{E_{0}+\Delta E / 2} d E G(E, r), \\
& u_{2}(r)=\frac{1}{\Delta E} \int_{E_{0}-\Delta E / 2}^{E_{0}+\Delta E / 2} d E F(E, r),
\end{aligned}
$$


where $E_{0}$ is the energy of the state which is described by the functions $u_{1}(r)$ and $u_{2}(r)$. Equations (62) imply the normalization

$$
\int_{0}^{\infty} d r\left[u_{1}^{2}(r)+u_{2}^{2}(r)\right]=1
$$

which is evidently of the same type as Eq. (60) for the bound states. This is possible, because these wave packets fall off as $1 / r$ in the asymptotic region, where the amplitude of the oscillation is dampened out as $r \rightarrow \infty$. All numerical intergrations were performed by Gaussian quadrature. The radial matrix elements from Eq. (40) exhibit the general structure

$$
\begin{aligned}
I & =\left(\beta_{1} \beta_{3}\left\|v_{L}\right\| \beta_{2} \beta_{4}\right) \\
& \sim \int_{0}^{\infty} d r_{1} \int_{0}^{\infty} d r_{2} R_{\kappa_{1}}^{\dagger}\left(r_{1}\right) R_{\kappa_{2}}\left(r_{1}\right) \frac{r_{<}^{L}}{r_{>}^{L+1}} R_{\kappa_{3}}^{\dagger}\left(r_{2}\right) R_{\kappa_{4}}\left(r_{2}\right),
\end{aligned}
$$

where the functions $R_{\kappa}(r)$ are the two-component radial functions defined in Eq. (37). Although the integration kernel formally is not separable, one can express these matrix elements as products of one-dimensional integrals:

$$
\begin{aligned}
I \sim \int_{0}^{\infty} d r_{1}\left\{P_{\kappa_{1} \kappa_{2}}\left(r_{1}\right)[\right. & \int_{0}^{r_{1}} d r_{2} \frac{r_{2}^{L}}{r_{1}^{L+1}} P_{\kappa_{3} \kappa_{4}}\left(r_{2}\right) \\
& \left.\left.+\int_{r_{1}}^{\infty} d r_{2} \frac{r_{1}^{L}}{r_{2}^{L+1}} P_{\kappa_{3} \kappa_{4}}\left(r_{2}\right)\right]\right\},
\end{aligned}
$$

where $P_{\kappa_{i} \kappa_{j}}(r)=G_{\kappa_{i}}(r) G_{\kappa_{j}}(r)+F_{\kappa_{i}}(r) F_{\kappa_{j}}(r)$. This allowed us to apply fast and highly accurate GaussLegendre integration routines.

\section{DISCUSSION OF THE RESULTS}

After reviewing the theoretical framework for the description of correlation effects in the QED vacuum we now present various numerical results for superheavy quasiatoms. In addition to the equation (42) for the effective interaction we have solved the RPA equations (46) directly, since this constitutes an essential test of the field-theoretical methods used in the present work as well as of the numerical computations. The solutions of these equations also provide the $p$ - $h$ amplitudes, which are needed for the evaluation of radiative transition strengths including correlation effects.

We begin the discussion with the single-particle wave functions which constitute our basis set. In Fig. 6 we compare the radial density distribution $\rho r^{2}$ of a $K$-shell electron in the Coulomb field of a lead nucleus $(Z=82)$ with that for the superheavy atom with $Z=169$. The increase of the nuclear charge causes a striking localization of the $1 s$ wave functions within one Compton wavelength of the electron, while the maximum of $\rho r^{2}$ is located in the vicinity of the nucleus. For large nuclear charge numbers the so-called small component $F$ exhibits the same magnitude as the large component $G$.

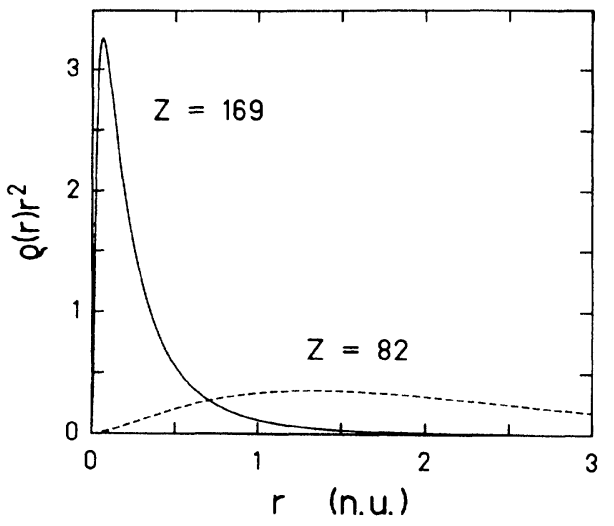

FIG. 6. Radial densities of a $K$-shell electron in hydrogenlike systems with $Z=82$ and 169 , respectively, as functions of the radial coordinate $r$. Natural units $(\hbar=c=m=1)$ are employed.

In Figs. 7(a) and 7(b) we display the radial components of the $s$ continuum wave function $(\kappa=-1)$ for $Z=169$ with the energies $E=2.15$ and -2.15 , respectively. The sine and cosine behavior is obvious. Note that for the negative-energy state $(E=-2.15)$ the small component $F$ exceeds in magnitude the large component $G$, in con-
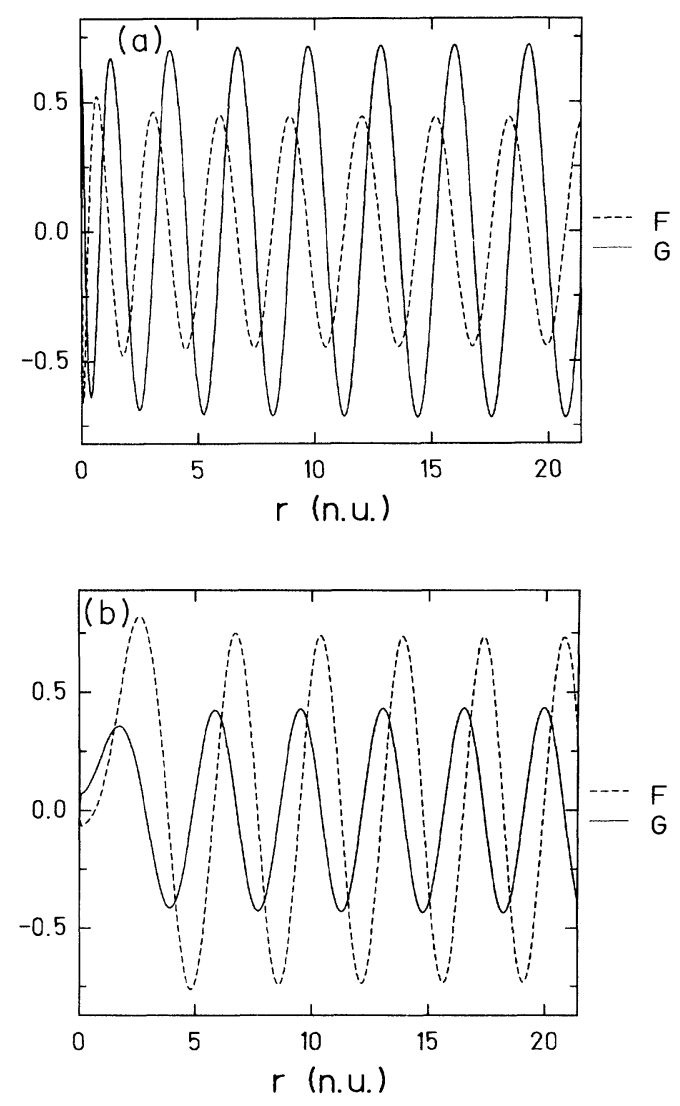

FIG. 7. Radial continuum wave functions $(\kappa=-1)$ for $Z=169$ and for the energies (a) $E=2.15 \mathrm{~m}$ and (b) $E=$ $-2.15 m$, respectively. Natural units $(\hbar=c=m=1)$ are employed. 
trast to the positive-energy state. We note that Coulomb distortion effects are more important for negative-energy states [Fig. 7(b)].

In Fig. 8 the $s$ wave-packet components $u_{1}$ and $u_{2}$ are depicted as functions of the radial coordinate $r$. We have chosen the same parameters as in Fig. 7 in order to point out the striking difference between a wave packet and a continuum wave function. The characteristic shape of a wave packet is determined by the superposition of monochromatic waves according to Eqs. (61). The integrations on the energy scale were performed numerically with a Gaussian quadrature. The number of grid points was varied in order to achieve an accurate error control. From Fig. 8(a) it is evident that the integration over the continuum wave functions damps out the amplitude of the oscillations leading to a typical $1 / r$ decline of the wave packet. In Fig. 8(b) we have plotted the radial density $\rho r^{2} \sim u_{1}^{2}+u_{2}^{2}$ for the wave packet describing the negative-energy $s$ state with $E=-2.3$. The positrons experience a strong Coulomb repulsion, in contrast to the electrons which are attracted by the nuclear potential.

Having calculated the single-particle wave functions we are able to evaluate the matrix elements of the bare $p-h$
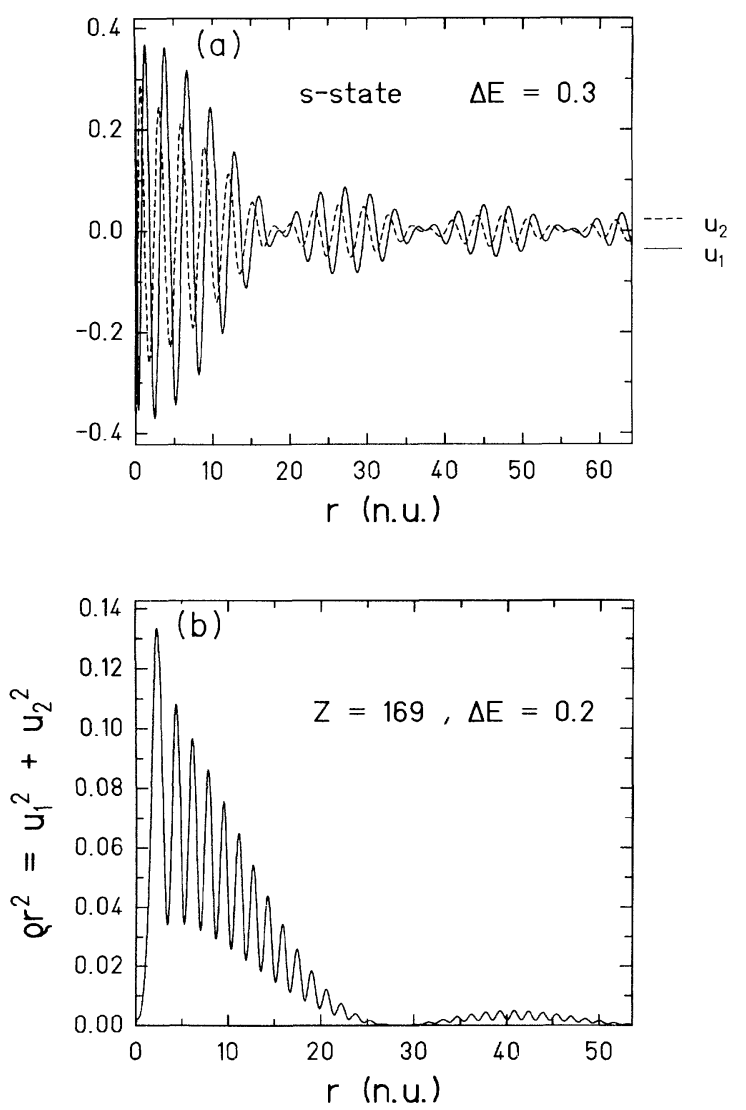

FIG. 8. Relativistic wave-packet components (a) and radial density (b), respectively, for $s$ states depending on the radial coordinate $r$. (a) $E=2.15 \mathrm{~m}$ and $\Delta E=0.3 \mathrm{~m}$, (b) $E=-2.3 m$ and $\Delta E=0.2 m$. Natural units $(\hbar=c=m=1)$ are employed.

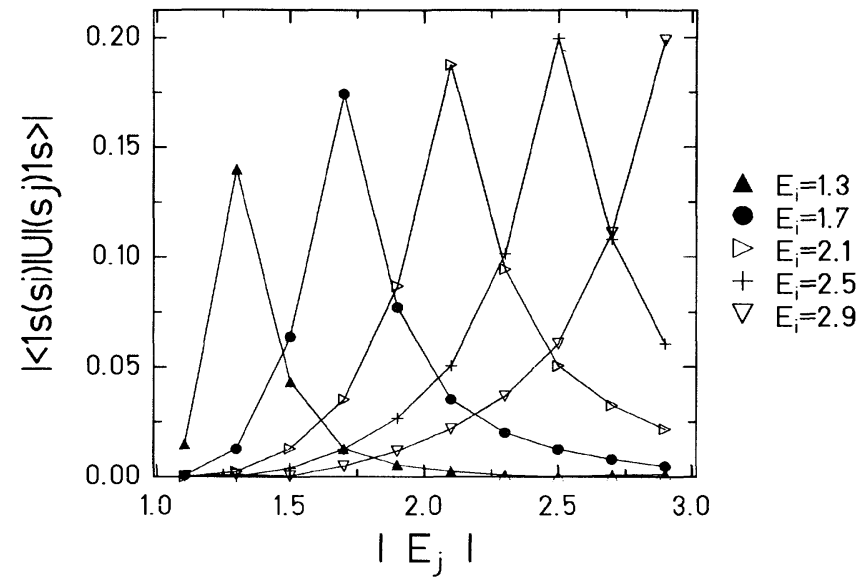

FIG. 9. Radial matrix elements depending on the energy of one of the continuum states. Both involved bound states correspond to $1 s$ wave functions.

interaction from Eq. (40). The numerical computations were performed by the method described in the preceding section. In Fig. 9 several bound-free matrix elements are plotted as function of the energy of one of the two involved continuum states. The bound wave function is the $K$-shell wave function of the system with $Z=169$, corresponding to the eigenenergy $E_{1 s}=-0.957$. The continuum states are described by wave packets with a level spacing $\Delta E=0.2$, i.e., $\simeq 100 \mathrm{keV}$. First, we notice that generally these matrix elements attain their maximum value for diagonal elements $(i=j)$.

In Fig. 10 we depict the same type of matrix elements as in Fig. 9, except that the $2 s$ wave function was considered as bound state. Compared to Fig. 9 we observe no significant difference.

In contrast, Fig. 11 exhibits a quite different behavior of the matrix elements. Here we considered $1 s$ and $2 s$ wave functions, respectively, which are the off-diagonal elements of the determinant from Eq. (58) with (57), or

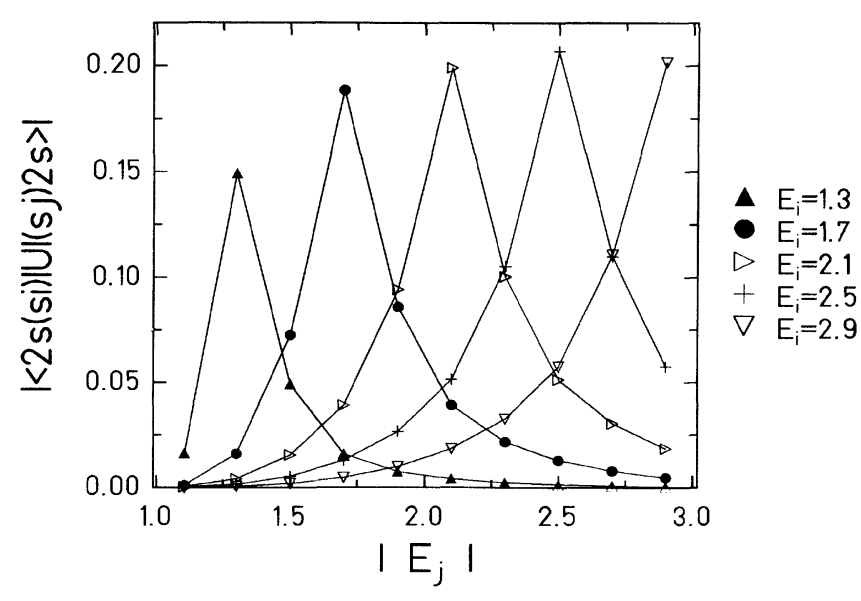

FIG. 10. The same as in Fig. 9. Both involved bound states correspond to $2 s$ wave functions. 


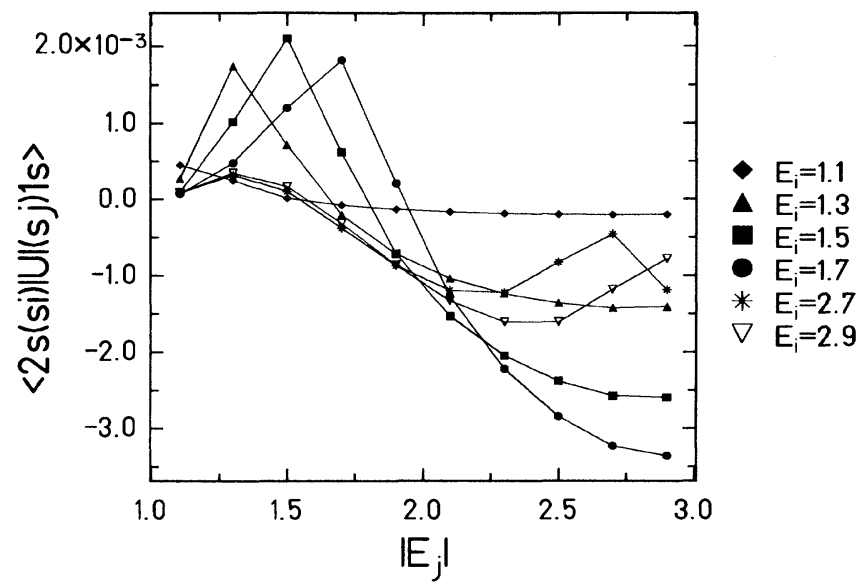

FIG. 11. The same as in Figs. 9 and 10. $2 s$ and $1 s$ bound states are involved.

alternatively of the RPA matrix from Eqs. (46). The first important feature which we want to point out is that these matrix elements are at least three orders of magnitude smaller than the diagonal matrix elements from Figs. 9 and 10, respectively. In addition, they display a more or less oscillatory behavior as functions of the continuum energy. This large difference between the magnitudes of the diagonal and off-diagonal elements will have important implications on the amount of mixing of the $p$ - $h$ configurations.

In Table I the excitation energies of the system with $Z=169$ are listed. We considered the $L=0$ component of Eq. (42) and thus investigated the possible existence of a monopole excitation mode. The $1 s$ and $2 s$ states were taken above the Fermi level, while 15 wave packets describing continuum states were taken into account. The width of the wave packets was taken as $\Delta E=0.2$, such that the deepest lying hole state corresponds to the energy $E_{h}=-3.9$, or approximately $-2 \mathrm{MeV}$. The first

TABLE I. Excitation energies $\omega$ taking into account the $p-h$ interaction and unperturbed $p-h$ energies $E_{p}-E_{h}$ in natural units.

\begin{tabular}{cccc}
\hline \hline$E_{p}-E_{h}$ & $\omega$ & $E_{p}-E_{h}$ & $\omega$ \\
\hline 0.148 & 0.1485 & 2.343 & 2.3444 \\
\hline 0.343 & 0.3440 & 2.345 & 2.3464 \\
\hline 0.543 & 0.5442 & 2.543 & 2.5443 \\
\hline 0.743 & 0.7443 & 2.545 & 2.5465 \\
\hline 0.943 & 0.9443 & 2.743 & 2.7444 \\
\hline 1.143 & 1.1444 & 2.745 & 2.7465 \\
\hline 1.343 & 1.3444 & 2.943 & 2.9442 \\
\hline 1.543 & 1.5445 & 2.945 & 2.9465 \\
\hline 1.550 & 1.5505 & 3.145 & 3.1465 \\
\hline 1.743 & 1.7445 & 3.345 & 3.3465 \\
\hline 1.745 & 1.7461 & 3.545 & 3.5464 \\
\hline 1.943 & 1.9445 & 3.745 & 3.7465 \\
\hline 1.945 & 1.9463 & 3.945 & 3.9462 \\
\hline 2.143 & 2.1444 & 4.145 & 4.1467 \\
\hline 2.145 & 2.1464 & 4.345 & 4.3463 \\
\hline \hline
\end{tabular}

column contains the unperturbed particle-hole energies, while the second one lists the corresponding excitation energies with $p$ - $h$ correlations taken into account as resulting from the numerical solution of the Bethe-Salpeter equation for $W(\omega)$. We have also solved the RPA eigenvalue equations in order to check the numerical computations. From Table I it can be seen that the shifts of the energies due to the $p$ - $h$ interaction are rather negligible. The largest relative difference between the free and correlated $p$ - $h$ excitation energies is smaller than $0.3 \%$. It is worthwhile to remark that with increasing absolute value of the positron energy, the relative energy shifts decrease steadily. For the deepest lying negative-energy state the relative modification of the energy shift amounts only to about $0.03 \%$. All unperturbed energies are shifted by less than $1 \mathrm{keV}$. This implies that there is no indication that one or more states of the interacting system are formed as a coherent superposition of many single-particle excitations, in which each excitation has about equal weight.

In order to achieve a better understanding of the typical collective behavior, we have also considered a schematic model [21]. Here all matrix elements are set $a d$ $h o c$ equal to a common value $\beta$. As a major advantage all calculations can be carried out analytically.

In Fig. 12 we have depicted the determinant from Eq.
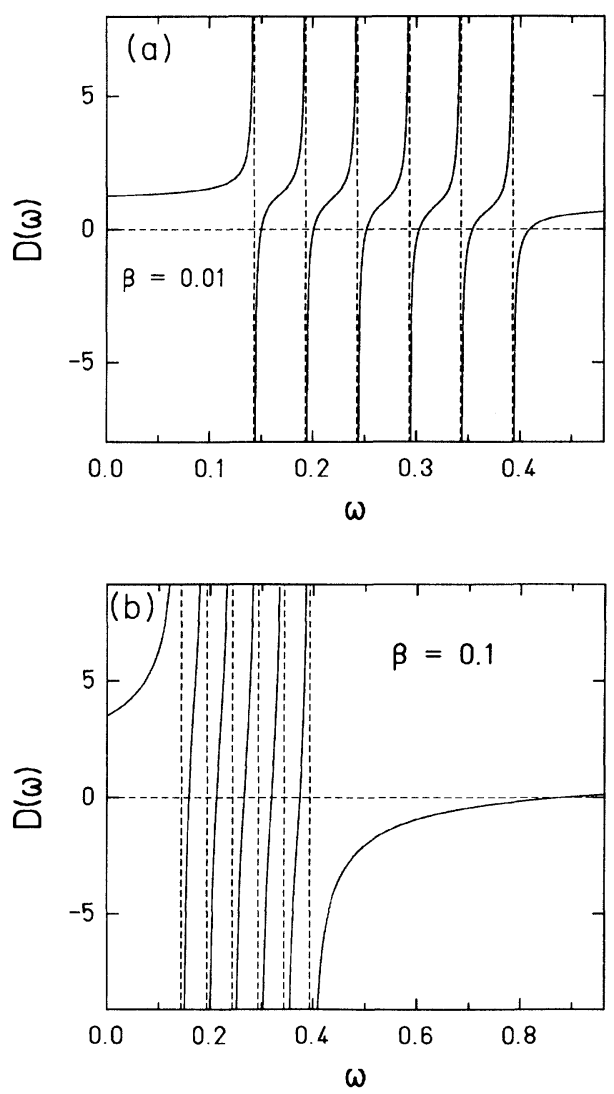

FIG. 12. The determinant from Eq. (58) as a function of the frequency $\omega$ in the case of the schematic model. All interaction matrix elements are chosen to be (a) $\beta=0.01$ and (b) $\beta=0.1$, respectively. 
(58) as a function of the frequency $\omega$ for this special case. We have considered only one particle state with energy $E_{p}=-0.957$ and six hole states with energies $E_{h}=-1.1$, $-1.15,-1.2,-1.25,-1.3$, and -1.35 , respectively. The intersections of the dashed vertical lines with the horizontal line passing through zero represent the positions of the unperturbed excitation energies $E_{p}-E_{h}$, while the intersections of the full lines with the same horizontal line provide the corresponding shifts which are a consequence of the $p$ - $h$ interaction. These are exactly the roots of the secular equation (58). From Fig. 12(a), where we considered the situation with $\beta=0.01$, it can be deduced that the different excitation energies are shifted by about the same amount of magnitude. This behavior is changed drastically in Fig. 12(b), where we fixed $\beta=0.1$. It is evident that one particular level is pushed to a much higher energy than the other levels. The corresponding state displays a collective character, i.e., it carries contribution from all other single-particle excitations which are involved with practically equal weight in its formation. One can understand the existence of such a collective state in terms of the relative difference between the diagonal and off-diagonal matrix elements of the interaction matrix, since it is just the magnitude of the off-diagonal terms which is responsible for the amount of mixing of the different $p$ - $h$ configurations. For our system under investigation the off-diagonal elements are about three orders of magnitude smaller than the diagonal elements, demonstrating that correlation effects are of minor importance for the $Q E D$ vacuum.

At this point we would like to mention that in Refs. $[22,23]$ it has been suggested that QED may possess a new vacuum state which can be formed in heavy-ion collisions. Indeed, it has been demonstrated in Refs. [24, 25] that QED has a phase in which chiral symmetry is broken, provided the coupling constant $\alpha$ exceeds the critical value $\alpha_{c} \simeq \pi / 3$. According to Ref. [26] the critical value of $\alpha$ depends on the electric charge number $Z$. Thus, we have also considered the possibility that collective states can be formed for a QED coupling constant which is greater than its value in the real world. The purpose of the discussion of this phase transition in the context of our work is solely to motivate our calculation with a coupling constant which is greater than its value in conventional QED. Of course, this calculation has no implications on the QED phase transition. The question which we want to study at this point is whether a QED coupling constant greater than its value of $\alpha \simeq \frac{1}{137}$ allows for the formation of collective excited states of the Dirac vacuum. In Table II we present the energy shifts caused by the $p$ - $h$ interaction for the two values of the coupling constant $\alpha=0.1<\alpha_{c}$ for $Z=1$ and $\alpha=1.11>\alpha_{c}$ for $Z=13$, respectively. These results demonstrate that except for the fact that all excitation energies are shifted by a larger amount, which is proportional to the modification of $\alpha$, the situation remains just the same as before, i.e., there is no indication concerning the existence of collective states.

In the remaining part of this section we would like to present our results concerning the dimensionless transition strengths $\bar{f}_{L}$ with correlations. In order to check
TABLE II. Excitation energies $\omega$ and corresponding unperturbed $p$ - $h$ energies $E_{p}-E_{h}$ for different values of the coupling constant (a) $\alpha=0.1$ and (b) $\alpha=1.11$, respectively.

\begin{tabular}{ccc}
\hline \hline$E_{p}-E_{h}$ & $\omega(\alpha=0.1)$ & $\omega(\alpha=1.11)$ \\
\hline 0.148 & 0.155 & 0.222 \\
\hline 0.343 & 0.357 & 0.490 \\
\hline 0.543 & 0.559 & 0.711 \\
\hline 0.743 & 0.760 & 0.923 \\
\hline 0.943 & 0.961 & 1.131 \\
\hline 1.143 & 1.162 & 1.338 \\
\hline 1.343 & 1.362 & 1.544 \\
\hline 1.543 & 1.557 & 1.627 \\
\hline 1.550 & 1.563 & 1.750 \\
\hline 1.743 & 1.760 & 1.899 \\
\hline 1.745 & 1.764 & 1.956 \\
\hline 1.943 & 1.963 & 2.124 \\
\hline 1.945 & 1.964 & 2.271 \\
\hline 2.145 & 2.164 & 2.338 \\
\hline 2.345 & 2.346 & 2.547 \\
\hline 2.545 & 2.565 & 2.753 \\
\hline 2.745 & 2.765 & 2.958 \\
\hline 2.945 & 2.966 & 3.164 \\
\hline 3.145 & 3.166 & 3.368 \\
\hline 3.345 & 3.366 & 3.674 \\
\hline \hline
\end{tabular}

our numerical integration routines, first we computed electronic transition amplitudes of multipolarities $M 1$, $M 2$, and $E 1$, respectively, which we have compared with previous work. The systems with charges $Z=82,145$, and 164 were considered, respectively. Our results agree within $15 \%$ with those presented in Ref. [27], where

TABLE III. Dimensionless strengths for $M 1$ transitions for $Z=169$. $f_{L}(m)$ are the bare strengths, while $\bar{f}_{L}(m)$ include particle-hole correlations.

\begin{tabular}{ccc}
\hline \hline & & \\
Transition $(M 1)$ & $f_{1}(\mathrm{~m})$ & $\bar{f}_{1}(\mathrm{~m})$ \\
\hline$s_{1} \rightarrow 1 s$ & $2.682 \times 10^{-8}$ & $2.633 \times 10^{-8}$ \\
\hline$s_{2} \rightarrow 1 s$ & $5.511 \times 10^{-6}$ & $5.475 \times 10^{-6}$ \\
\hline$s_{3} \rightarrow 1 s$ & $3.057 \times 10^{-5}$ & $2.633 \times 10^{-5}$ \\
\hline$s_{4} \rightarrow 1 s$ & $8.154 \times 10^{-5}$ & $8.084 \times 10^{-5}$ \\
\hline$s_{5} \rightarrow 1 s$ & $1.584 \times 10^{-4}$ & $1.573 \times 10^{-4}$ \\
\hline$s_{6} \rightarrow 1 s$ & $2.475 \times 10^{-4}$ & $2.462 \times 10^{-4}$ \\
\hline$s_{7} \rightarrow 1 s$ & $3.448 \times 10^{-4}$ & $3.440 \times 10^{-4}$ \\
\hline$s_{8} \rightarrow 1 s$ & $4.512 \times 10^{-4}$ & $4.539 \times 10^{-4}$ \\
\hline$s_{9} \rightarrow 1 s$ & $5.538 \times 10^{-4}$ & $5.552 \times 10^{-4}$ \\
\hline$s_{10} \rightarrow 1 s$ & $6.188 \times 10^{-4}$ & $6.195 \times 10^{-4}$ \\
\hline$s_{1} \rightarrow 2 s$ & $1.497 \times 10^{-9}$ & $1.362 \times 10^{-9}$ \\
\hline$s_{2} \rightarrow 2 s$ & $9.310 \times 10^{-6}$ & $9.411 \times 10^{-6}$ \\
\hline$s_{3} \rightarrow 2 s$ & $3.610 \times 10^{-5}$ & $3.541 \times 10^{-5}$ \\
\hline$s_{4} \rightarrow 2 s$ & $6.441 \times 10^{-5}$ & $6.390 \times 10^{-5}$ \\
\hline$s_{5} \rightarrow 2 s$ & $8.515 \times 10^{-5}$ & $8.475 \times 10^{-5}$ \\
\hline$s_{6} \rightarrow 2 s$ & $9.688 \times 10^{-5}$ & $9.661 \times 10^{-5}$ \\
\hline$s_{7} \rightarrow 2 s$ & $1.007 \times 10^{-4}$ & $1.005 \times 10^{-4}$ \\
\hline$s_{8} \rightarrow 2 s$ & $1.005 \times 10^{-4}$ & $1.006 \times 10^{-4}$ \\
\hline$s_{9} \rightarrow 2 s$ & $9.591 \times 10^{-5}$ & $9.626 \times 10^{-5}$ \\
\hline$s_{10} \rightarrow 2 s$ & $8.411 \times 10^{-5}$ & $9.211 \times 10^{-5}$ \\
\hline \hline
\end{tabular}


Dirac-Hartree-Fock wave functions have been utilized, in contrast to the Coulomb wave functions employed in this work.

In Table III we present dimensionless transition strengths for electronic transitions of multipolarity $M 1$ from $s$ states below the Fermi level into the $1 s$ and $2 s$ bound states for $Z=169$, respectively. The first column contains the specific transition, while in the second and third columns we list our results for the quantities $f_{L}(m)$ (bare strengths) and $\bar{f}_{L}(m)$ (with the inclusion of correlations) from Eqs. (52), (54), and (55), respectively. It can be concluded from this table that the $p$ - $h$ interaction has only a minor influence on the bare transition strengths. The relative difference between $f_{L}(m)$ and $\bar{f}_{L}(m)$ does not exceed $10 \%$, and in most cases is of the order $1 \%$. Consequently, these results support our conclusion concerning the minor importance of correlations in the QED vacuum of superheavy quasiatoms.

\section{CONCLUSIONS}

As a major objective of our investigation we examined the possibility that the QED ground state in the field of a large charge possesses collective excitation modes, which would be similar to those encountered in various many-particle systems. The Green's function technique has proven to be a powerful tool for the exploration of collective effects in interacting many-body systems with a wide range of applicability. Our treatment incorporates the same physical ingredients as the standard randomphase approximation.

From the results presented in the preceding section we deduce that the $p$ - $h$ correlations display only a minor effect on the electron-positron excitation energies in the field of a superheavy nucleus. The shifts of the energies do not exceed $1 \mathrm{keV}$ and the relative difference between the energies of the free and interacting $p$ - $h$ configurations is smaller than $0.5 \%$. We found no indication for the existence of collective excited states. Our results concerning the transition strengths with and without the inclusion of the $p-h$ interaction clearly support this conclusion. They provide a direct measure for the amount of mixing of the involved configurations, which was also found to be small.

The application of the RPA to the infinite electron gas and to nuclei yields collective states, i.e., states that represent coherent superpositions of many single-particle excitations with each excitation having about equal weight. This behavior is determined by the relative difference between the diagonal and off-diagonal elements which are involved in these systems. The unperturbed spectrum of a nucleus consists of a group of states which are closely spaced. Since correlations are rather important, strong mixing occurs with the consequence that collective states are formed. However, the excitation spectrum of the system we have investigated, i.e., the Dirac vacuum in the presence of a strong Coulomb field, is spread over the entire energy range, whereas the nuclear energy spectrum is bunched. Consequently, the existence of collective excitation modes in the $Q E D$ vacuum is, a priori, much less likely, and our calculation actually verifies this hypothesis.

\section{ACKNOWLEDGMENTS}

We are grateful for various exciting discussions with $\mathrm{J}$. Reinhardt and A. Schäfer. One of us (B.M.) acknowledges support by the U.S. Department of Energy (Grant No. DE-FG05-90ER40592) and thanks GSI for their hospitality.
* Present address: Bereich Schwerionenphysik, HahnMeitner-Institut Berlin, D-1000 Berlin 39, Germany.

[1] A. L. Fetter and J. D. Walecka, Quantum Theory of Many-Particle Systems (McGraw-Hill, New York, 1971).

[2] B. Müller, H. Peitz, J. Rafelski, and W. Greiner, Phys. Rev. Lett. 28, 1235 (1972).

[3] K. Smith, H. Peitz, B. Müller, and W. Greiner, Phys. Rev. Lett. 32, 554 (1974).

[4] W. Greiner, B. Müller, and J. Rafelski, Quantum Electrodynamics of Strong Fields (Springer, Heidelberg, 1985).

[5] P. Nozières, Theory of Interacting Fermi Systems (Benjamin, New York, 1964).

[6] A. B. Migdal, Theory of Finite Fermi Systems and Application to Atomic Nuclei (Wiley, New York, 1967).

[7] R. H. Lemmer and W. Greiner, Phys. Lett. 162B, 247 (1985).

[8] C. Itzykson and B. Zuber, Quantum Field Theory (Mc Graw-Hill, New York, 1980).

[9] N. Nakanishi, Suppl. Prog. Theor. Phys. 43, 1 (1969).

[10] G. Breit, Phys. Rev. 34, 553 (1929).

[11] G. Breit, Phys. Rev. 36, 383 (1930).

[12] W. R. Johnson, C. D. Lin, K. T. Cheng, and C. M. Lee, Phys. Scr. 21, 409 (1980).

[13] S. S. Schweber, An Introduction to Relativistic Quantum
Field Theory (Harper \& Row, New York, 1961).

[14] M. E. Rose, Relativistic Electron Theory (Wiley, New York, 1961).

[15] J. D. Jackson, Classical Electrodynamics (Wiley, New York, 1962).

[16] P. L. O. von Ross, Phys. Rev. 128, 911 (1962).

[17] H. A. Bethe and E. E. Salpeter, Quantum Mechanics of One and Two Electron Atoms (Springer, Berlin, 1957).

[18] J. H. Scofield, Phys. Rev. 179, 9 (1969).

[19] D. J. Thouless, Nucl. Phys. 22, 78 (1961).

[20] H. J. Bär and G. Soff, Physica 128C, 225 (1985).

[21] G. E. Brown and M. Bolsterli, Phys. Rev. Lett. 3, 472 (1959).

[22] L. S. Celenza, V. K. Mishra, C. M. Shakin, and K. F. Liu, Phys. Rev. Lett. 57, 55 (1986).

[23] L. S. Celenza, C. R. Ji, and C. M. Shakin, Phys. Rev. D 36, 2144 (1987).

[24] J. Kogut, E. Dagotto, and A. Kocic, Phys. Rev. Lett. 60, $772(1988)$

[25] V. Miransky, Nuovo Cimento A 90, 149 (1985).

[26] E. Dagotto and H. W. Wyld, Phys. Lett. 205, 73 (1988).

[27] G. Soff and B. Müller, Z. Phys. A 280, 243 (1977). 